\title{
A Hydraulic Capture Application for Optimal Remediation Design*
}

K. R. Fowler ${ }^{\mathrm{a}}$, C. T. Kelley ${ }^{\mathrm{b}}$, C. E. Kees ${ }^{\mathrm{b}}$, and Cass T. Miller ${ }^{\mathrm{c}}$

${ }^{a}$ Center for the Environment, Department of Mathematics and Computer Science, Clarkson University

Potsdam, NY 13699-5815, USA

${ }^{\mathrm{b}}$ Center for Research and Scientific Computing, Department of Mathematics, North Carolina State University

Raleigh, NC 27695-8205, USA

${ }^{\mathrm{c}}$ Center for the Advanced Study of the Environment, Department of Environmental

Sciences and Engineering

University of North Carolina

Chapel Hill, NC 27599-7400, USA

The goal of a hydraulic capture model for remediation purposes is to design a well field so that the direction of groundwater flow is altered, thereby halting or reversing the migration of a contaminant plume. Management strategies typically require a well design that will contain or shrink a plume at minimum cost. Objective functions and constraints can be nonlinear, non-convex, non-differentiable, or even discontinuous. The solution uses optimization algorithms with groundwater flow and possibly transport simulators. The formulation of the objective function dictates possible optimization algorithms that can be used. For example, a gradient based method is likely to fail on a discontinuous objective function or gradient information may not be available. Computational efficiency as well as accuracy is desirable and often influences the choice of solution method. In this paper we present two hydraulic capture models. Our motivation is a hydraulic capture application proposed in the literature for benchmarking purposes. We present numerical results for the three models using the implicit filtering algorithm.

\section{INTRODUCTION}

Hydraulic capture methods for remediation attempt to control the direction of groundwater movement in order to manipulate the migration of a contaminant plume. A well field can be designed, for example, so that gradients point towards the interior of the plume, resulting in containment and possible shrinkage of the plume. Plume migration control is an active area of research in environmental engineering. Research is directed at 1) improving models of subsurface flow and transport, 2) incorporating installation

\footnotetext{
${ }^{*}$ This research was partially supported by National Science Foundation grant \#DMS-0070641k, \#DMS0209695, and \#DMS-0112542, and Army Research Office grants \#DAAD19-02-1-0391 and \#DAAD19-021-0111. The research by KRF was also supported by a US Department of Education GAANN fellowship
} 
and operational costs of $\mathrm{HC}$ into the objective functions, and 3) developing and calibrating capture constraints so that computational efficiency is obtained in the modeling process while simultaneously avoiding excessive pumping in the final well design. Once an objective function and constraints are developed, optimization techniques are used to determine the well design that minimizes the remediation cost while satisfying the constraints. Mathematically, these problems are challenging in that simulators must work in conjunction with optimization algorithms-evaluation of the objective function and constraints typically requires a simulation. This so-called black-box formulation means that necessary gradient information may be difficult or impossible to obtain.

Our motivation is a hydraulic capture application proposed in the literature as part of a suite of benchmarking test problems [10]. In [10] the authors address many of the existing challenges in optimal design problems involving saturated flow and transport, which includes the lack of representative problems to be used for comparison purposes. The test problems were developed after an extensive literature search and are indicative of real world problems. The problems are described in [11] as is data for several different physical domains ranging from homogeneous, confined aquifers to complicated, unconfined aquifers with hydraulic conductivities that are represented using correlated random fields corresponding to typical values observed in nature. Objective functions and constraints are specified for all the problems except the hydraulic capture application-it is left to the modeler to choose the capture constraint and the concentration contour of the plume boundary. This test problem is an opportunity to effectively compare a variety of constraint formulations and optimization approaches. Guidelines for comparison are outlined in [10].

The objectives of this paper are: (1) to describe three formulations of the HC problem in [10], (2) to compare the two formulations using a sampling method for optimization, and (3) point the way towards future studies for these applications.

We proceed by describing the three $\mathrm{HC}$ formulations in $\S 2$, describing the benchmarking problem in $\S 3$, presenting numerical results in $\S 4$ and we conclude and discuss future work in $\S 5$.

\section{HYDRAULIC CAPTURE MODELS}

We will consider two hydraulic capture models; flow-based hydraulic control (FBHC) and transport-based concentration control (TBCC). For both models, the optimization problem is posed as

$\min _{\mathbf{u} \in \Omega} J(\mathbf{u})$

where $\mathbf{u}(\mathbf{w})$ is a vector of decision variables that depends on a vector $\mathbf{w}$ of state variables, such as fluid pressures or species concentrations. The decision variables can be integer or real valued such as the number of wells (integer valued), extraction or injection rates, and well locations (real valued). The state variables represent the state of the physical system given a set of decision variables and are determined by solving the system of equations that model fluid flow and contaminant transport. In (1), $\Omega$ is the feasible region of $\mathbf{u}$ and is represented by a set of constraint equations. Typically, $J(\mathbf{u})$ is a function that represents the cost to operate a set of wells and may include an installation and maintenance cost. 
The objective function $J(\mathbf{u})$ can include more than one function to be minimized, as in the case in multi-objective optimization. In a multi-objective problem, there may be one function to minimize the cost and another to maximize clean-up.

Evaluation of $J(\mathbf{u})$ or the constraints that define $\Omega$ require the solution to the equations that model saturated flow and possibly contaminant transport. The model for saturated flow is given by

$S_{s} \frac{\partial h}{\partial t}=\nabla \cdot(\mathbf{K} \cdot \nabla h)+\bar{S}$,

where $S_{s}(1 / m)$ is the specific storage coefficient, $h(m)$ is the hydraulic head, $\mathbf{K}(m / s)$ is the hydraulic conductivity tensor, and $\bar{S}\left(\mathrm{~m}^{3} / \mathrm{s}\right)$ is a fluid source term and is where the decision variables enter the state equation for the $\mathrm{HC}$ problem. The equation for contaminant transport is given by

$\frac{\partial\left(\theta^{\alpha} C^{\iota}\right)}{\partial t}=\nabla \cdot\left(\theta^{\alpha} \mathbf{D}^{\iota} \cdot \nabla C^{\iota}\right)-\nabla \cdot\left(\mathbf{q} C^{\iota}\right)+I^{\iota}+R^{\iota}+S^{\iota}$

where $C^{\iota}\left(\mathrm{kg} / \mathrm{m}^{3}\right)$ is the concentration of species $\iota$ in the aqueous phase, $\theta^{\alpha}$ is the volume fraction of the aqueous phase, $\mathbf{D}$ is a hydrodynamic dispersion tensor, $\mathbf{v}(\mathrm{m} / \mathrm{s})$ is the mean pore velocity, $\mathbf{q}(\mathrm{m} / \mathrm{s})$ is the Darcy velocity, and $I^{\iota}, R^{\iota}, S^{\iota}$ represent interphase mass transfer, biogeochemical reactions, and source of mass respectively.

In HC models, decision variables are typically a subset of the following: $n$, the number of wells in the design with $n \leq N$, where $N$ is the number of candidate wells, $\left\{Q_{i}\right\}_{i=1}^{N}\left(m^{3} / s\right)$ the possible extraction or injection rates at the wells, and $\left\{\mathbf{x}_{i}\right\}_{i=0}^{N}(m)$, the locations of the wells. Constraints typically include bounds on the well capacities and the hydraulic head at each well location given by

$$
\begin{aligned}
& Q^{\text {emax }} \leq Q_{i} \leq Q^{i m a x}\left(m^{3} / s\right), i=1, \ldots, n \\
& h^{\text {max }} \geq h_{i} \geq h^{\text {min }}(m), i=1, \ldots, n
\end{aligned}
$$

where, $Q^{\text {emax }}$ is the maximum extraction rate at any well, $Q^{\text {imax }}$ is the maximum injection rate at any well, $h^{\max }$ is the maximum allowable head, and $h^{\min }$ is the minimum allowable head. Note that evaluation of (5) requires a solution to (2).

\subsection{FLOW-BASED HYDRAULIC CONTROL}

Flow-based hydraulic control is a technique for plume containment that enforces head gradient constraints around the perimeter of the plume. We consider two formulations of this approach and refer to them as FBHC1 and FBHC2. For FBHC1, a head gradient constraint can be formulated as a constraint on the difference in hydraulic head values at specified locations, for example

$h_{1}^{k}-h_{2}^{k} \geq d(m), k=1 \ldots M$

where $h_{1}, h_{2}$ are hydraulic head values at specified nodes for each $k$ and $d$ is the bound on the difference. Here $M$ is the number of head gradient constraints imposed around the boundary. The constraint (6) can be used to enforce head gradients vertically or 
horizontally. For example, in the simple case where $h_{1}$ are $h_{2}$ are aligned at a distance of distance $\Delta x$ apart, then dividing (6) by $\Delta x$ yields

$$
\left(\frac{h_{1}^{k}-h_{2}^{k}}{\Delta x}\right) \geq \frac{d}{\Delta x}(m / s) .
$$

For FBHC1, $J(u)$ is minimized subject to (4), (5), and (6). This method is attractive in that only a solution to (2) is required to evaluate the constraints (5),

One of the main drawbacks of the FBHC1 approach is that the constraint (6) must be calibrated to ensure the plume is captured and to avoid excessive pumping [12]. To better define the capture zone, another approach (FBHC2) is to enforce that the head gradient at a control point located on or just outside the plume boundary points towards an extraction well. This can be imposed in the $x$ direction, for example, as

$\hat{h}_{x}^{k, j} \geq d, k=1 \ldots M, j \in\left\{1,2, \ldots, n^{e}\right\}$.

Here $M$ is the number of control points, $n^{e}$ is the number of extraction wells, and $\hat{h}_{x}^{k, j}$ is a numerical directional derivative at the $k$ th control point toward extraction well $j$. The constraint is satisfied if each component of the head gradient at a control point is in the direction of at at least one extraction well. For FBHC2, $J(u)$ is minimized subject to (4), (5), and (8).

\subsection{TRANSPORT-BASED CONCENTRATION CONTROL}

A direct approach for plume containment is to impose constraints on the concentration at specified locations. This constraint can be expressed as

$C_{j} \leq C_{j}^{\max }\left(\mathrm{kg} / \mathrm{m}^{3}\right)$

where $C_{j}$ is the concentration at some observation node $j$ and $C_{j}^{\max }$ is the maximum allowable concentration. This is often enforced at the final time, but can also vary with time. Time varying pumping schemes have been developed for pump and treat applications and can lead to a more efficient well field design [13]. TBCC uses (9) with the constraints (4) and (5). Note that evaluation of (9) requires a solution to (3) in addition to (2), making the TBCC approach more computationally expensive. Moreover, objective functions and constraints involving concentrations are nonconvex in some situations, [3], [2], making the minimization problem more difficult.

\section{BENCHMARKING PROBLEM}

We now describe the HC problem proposed in [10]. The physical domain is a $1000 \times$ $1000 \times 30(\mathrm{~m})$, unconfined aquifer. Since the aquifer is unconfined, the head constraint, (5), depends nonlinearly on the pumping rates. There are five different hydraulic conductivity fields described in [10]. For this work we will use the simple homogeneous case with $K=5.01 \times 10^{-5}(\mathrm{~m} / \mathrm{s})$. Paired with the saturated flow equation, (2) we use the following boundary and initial conditions:

$\left.\frac{\partial h}{\partial x}\right|_{x=0}=\left.\frac{\partial h}{\partial y}\right|_{y=0}=\left.\frac{\partial h}{\partial z}\right|_{z=0}=0, t>0$ 
$q_{z}(x, y, z=h, t>0)=-1.903 \times 10^{-8}(\mathrm{~m} / \mathrm{s})$, where $q_{z}=-K \frac{\partial h}{\partial z}$,

$h(1000, y, z, t>0)=20-0.001 y(m)$ and $h(x, 1000, z, t>0)=20-0.001 x(m)$.

Here $q_{z}$ is the Darcy flux out of the domain, representing recharge into the aquifer that could be the result of rainfall. We use $h(x, y, z, 0)=h_{s}(m)$ as the steady state solution to the flow problem without wells. $S_{s}=2.0 \times 10^{-1}(1 / \mathrm{m})$ is the specific yield of the unconfined aquifer. The ground surface elevation is $z_{g s}=30(\mathrm{~m})$.

We simulate plume development using (3) from a finite source for five years with a constant concentration of $1 \mathrm{~kg} / \mathrm{m}^{3}$ located in the region bounded by $[(200,225) ;(475,525),(h, h-$ $2)](\mathrm{m})$. For this work, we choose the $5 \times 10^{-5}\left(\mathrm{~kg} / \mathrm{m}^{3}\right)$ contour line as the plume boundary and set $C_{j}^{\max }=5 \times 10^{-5}\left(\mathrm{~kg} / \mathrm{m}^{3}\right)$ in $(9)$.

\subsection{OBJECTIVE FUNCTION AND CONSTRAINTS}

We consider a capital (installation) cost $J^{c}$ and an operational cost $J^{o}$ and seek to minimize $J=J^{c}+J^{o}$. Note that a negative pumping rate means a well is extracting and a positive pumping rates means a well is injecting water. Our simulation time is $t_{f}=5$ years. The objective function, as proposed in $[10,11]$ is given by

$$
\begin{gathered}
J=\underbrace{\sum_{i=1}^{n} c_{0} d_{i}^{b 0}+\sum_{Q_{i}<0.0} c_{1}\left|Q_{i}^{m}\right|^{b_{1}}\left(z_{g s}-h^{m i n}\right)^{b_{2}}}_{J^{c}}+ \\
\underbrace{\int_{0}^{t_{f}}\left(\sum_{i, Q_{i}<0.0} c_{2} Q_{i}\left(h_{i}-z_{g s}\right)+\sum_{i, Q_{i}>0.0} c_{3} Q_{i}\right) d t}_{J^{o}}
\end{gathered}
$$

Where $c_{j}$ and $b_{j}$ are cost coefficients and exponents. Here $d_{i}=z_{g s}$ is the depth of well $i, Q_{i}^{m}$ is the design pumping rate and we use $Q_{i}^{m}= \pm 0.0064\left(\mathrm{~m}^{3} / \mathrm{s}\right)$ for this work, $h^{\text {min }}=10(\mathrm{~m})$ is the minimum allowable head, and $h_{i}(m)$ is the hydraulic head in well $i$. Injection wells are assumed to operate under gravity feed conditions. In $J^{c}$ the first term accounts for drilling and installing all the wells and the second term is an additional cost for pumps for the extraction wells. In $J^{o}$, the term pertaining the the extraction wells includes a lift cost to raise the water to the surface. The cost data are given in [11].

We include the number of wells, $n \leq N$, well locations $\left\{\left(x_{i}, y_{i}\right)\right\}_{i=1}^{n}$ and pumping rates $\left\{Q_{i}\right\}_{i=1}^{n}$ as decision variables. We assume all wells have a fixed depth, but the location can vary in the $x-y$ plane. For this work, if in the course of the optimization a well rate satisfies $\left|Q_{i}\right| \leq 10^{-6}\left(\mathrm{~m}^{3} / \mathrm{s}\right)$ we remove that well from the design space and do not include that well in the flow and transport simulation or cost calculation. Although this leads to a discontinuous objective function, the benefit is a decrease in the installation cost and a well design with fewer wells operating at higher rates.

For the constraints, in addition to $(4),(5)$ and the capture constraint, we constrain the net pumping rate with

$Q_{T}=\sum_{i=1}^{n} Q_{i} \geq Q_{T}^{\max }$

where $Q_{T}^{\max }\left(\mathrm{m}^{3} / \mathrm{s}\right)$ is the maximum allowable total extraction rate. 


\section{NUMERICAL RESULTS}

In this section we compare the FBHC methods to the TBCC method on the benchmarking problem. We use MODFLOW2000, [16], to solve (2) and MT3DMS, [15], to solve (3). MT3DMS was also used to generate the initial contaminant plume, as described in [11]. We discretize the domain on a $100 \times 100 \times 10$ grid and use a steady state solution to (2). We choose to enforce five head difference constraints, (6), for the FBHC1 approach with $d=10^{-4}$. For FBHC2 we impose gradients at four control points in (8) in both the $x$ and $y$ directions. Three control points are situated at the front of the plume and one is located down gradient from the plume to ensure the extraction well does not move outside of the plume boundary in the course of the optimization. We use five concentration constraints, (9), for the TBCC approach enforced in the same locations as (6) in the FBHC1 approach.

For optimization, we use a FORTRAN77 implementation of the implicit filtering algorithm called IFFCO (Implicit Filtering For Constrained Optimization) [4], [8]. The algorithm follows a projected quasi-Newton method that uses finite difference gradients and reduces the difference increment as the optimization progresses. Implicit filtering is a sampling method in that optimization is governed by function values and analytic gradients are not needed. The algorithm has successfully been applied to nonconvex, nonsmooth, and even discontinuous objective functions, including other applications from hydrology [3], [6].

IFFCO requires a feasible initial iterate. We start with two extraction and two injection wells for a total of $N=4$ candidate wells. We use $\pm Q_{i}=0.0064\left(\mathrm{~m}^{3} / \mathrm{s}\right)$ as the initial well rates. The cost at the initial iterate is $J\left(u_{0}\right)=\$ 78,587$. Table 1 shows the minimum cost found and the \%-decrease for both methods. The performance of a sampling method can be viewed in terms of how many function evaluations are needed to reach the optimal point. For this work, the expensive part of a function evaluation is a call to either MODFLOW2000 or MT3DMS. If (14) is not satisfied, a flow simulation was not performed in either approach and likewise if (5) was not satisfied then a transport simulation was not performed for the TBCC approach. The fourth column in Table 1 shows the number of times that MODFLOW2000 (mf2k) and MT3DMS (mt3d) were called for each approach. For both the FBHC and TBCC approach, the optimal design includes only one extraction well. The last column in Table 1 shows the optimal location and pumping rate of the one remaining well is the design.

\section{DISCUSSION}

- The numerical results were obtained on a PC running Linux Redhat 8.0 with a Pentium $42.53 \mathrm{Ghz}$ processor. A flow simulation took roughly 2 seconds while one transport simulation took anywhere between 45 and 55 seconds. The total run time was roughly 20 minutes or less for the FBHC approaches and 4.5 hours for the TBCC approach. The TBCC approach resulted in a slightly lower design cost than FBHC1 approach yet not

as low as the FBHC2 approach, and at a high computational expense. The FBHC2 approach results in a lower cost than the FBHC1 approach since the constraint (8) relates the gradient at points on the plume boundary to the extraction well locations, thereby better defining the capture zone. The optimal points found by all methods are very close 
Table 1

Numerical Results

\begin{tabular}{lllll}
\hline Method & $\begin{array}{l}\text { Cost } \\
(\$)\end{array}$ & \% Decrease & $\begin{array}{l}\text { Function } \\
\text { Calls }\end{array}$ & $\begin{array}{l}\text { Optimal Point } \\
(x, y) / Q\end{array}$ \\
\hline FBHC1 & $\$ 23,031$ & $70.7 \%$ & $395 \mathrm{mf} 2 \mathrm{k}$ & $(257,634)(\mathrm{m})$ \\
& & $0 \mathrm{mt} 3 \mathrm{~d}$ & $-0.0054\left(\mathrm{~m}^{3} / \mathrm{s}\right)$ \\
\hline FBHC2 & $\$ 21,560$ & $72.6 \%$ & $317 \mathrm{mf} 2 \mathrm{k}$ & $(250,650)$ \\
& & & $0 \mathrm{mt} 3 \mathrm{~d}$ & $-0.0039\left(\mathrm{~m}^{3} / \mathrm{s}\right)$ \\
\hline TBCC & $\$ 22,002$ & $72.0 \%$ & $312 \mathrm{mf} 2 \mathrm{k}$ & $(250,650)(\mathrm{m})$ \\
& & & $295 \mathrm{mt} 3 \mathrm{~d}$ & $-0.0043\left(\mathrm{~m}^{3} / \mathrm{s}\right)$ \\
\hline
\end{tabular}

in both well location and pumping rate and in all three cases, the plume was contained. We can conclude that for this simple, homogeneous case that the FBHC methods are sufficient. It is possible, however, that in the case of heterogeneities that the TBCC approach could result in significantly less pumping and a more efficient design since the plume boundary is precisely defined. Implementing the conductivity fields specified in [10], [11] is the subject of future work.

- To reduce run times, we recommend parallel optimization algorithms for these applications. Although these results were done in serial, a parallel version of IFFCO exists in which function evaluations and hence calls to the simulators are done on separate processors. The parallel version of IFFCO has been successfully applied to a well field design application, [6], also proposed in [10].

- Additional approaches for HC models have been developed, namely the particle-tracking based advective control approach. Here a particle tracking goundwater flow simulator is used and particle pathlines represent the movement of "parcels" of groundwater. If a particle is initialized on the plume boundary then remediation design is successful if the particle travels to an extraction well [12]. In [12], the authors compare the particle tracking approach to the FBHC approach and show that a more efficient remediation design can result. The advantage is that the plume boundary is clearly defined thereby avoiding the pitfall of overconstraining the head difference constraint in (6). The drawback is that the more particles that are used to represent the plume, the more computationally expensive the approach becomes. The application of this approach to the benchmarking problem, as well as other existing HC models, is one subject of our future work.

- This was a first attempt at solving the hydraulic capture problem proposed in [10] with both the FBHC and TBCC models. The formulation of the capture constraint is an open question and it is not clear which approach will work best given more complicated hydraulic conductivity fields. Moreover, future work includes investigating optimal choices for $d$ and constraint locations in (6) and (8).

- The formulation of the objective functions and constraints are nonlinear and discontinuous, making sampling methods a natural choice for optimization. The discontinuity is present in the installation cost due to the removal of a well from the design space if $\left|Q_{i}\right| \leq 10^{-6}$. Often, installation costs are ignored in remediation design problems in which 
operational costs can dominate for long remediation periods. The result is a continuous linear or nonlinear programming problem. In [9], the authors argue that for remediation periods less than ten years, inclusion of the installation cost can result in a more efficient design. The authors of [9] approximate the discontinuous installation cost with a continuous formulation that uses a penalty term. We direct the reader to [9] and the references therein for other continuous approximations of fixed costs. For this work, we were able to implement the original formulation of the objective function as specified in [10] since our choice of optimizer is equipped to deal with discontinuities. We did find however, that IFFCO was sensitive to the initial iterate and IFFCO and for several initial iterates, IFFCO would converge to a local minimum. Future work involves solving the HC benchmarking problem with a variety of sampling methods such as genetic algorithms, surrogate response surface approaches, and other stencil based methods to compare the performance of the optimization algorithms.

- Extensive reviews of hydraulic capture models exist in the literature (for example [1], [5], [14]) and a full survey of existing approaches is beyond the scope of this paper. We hope to give the reader a general overview of the problem as well as encourage interested modelers to investigate the benchmarking problem.

\section{REFERENCES}

1. David P. Ahlfeld and Manoutchehr Heidari, Applications of Optimal Hydraulic Control to Ground-Water Systems, Journal of Water Resources Planning and Management, 120(3) 350-365 (1994)

2. David P. Ahlfeld and Mary Palumbo Sprong, Presence of Nonconvexity in Groundwater Concentration Response Functions, Journal of Water Resources Planning and Management, 124(1) 8-14, (1998)

3. A. Battermann, J.M. Gablonsky, A. Patrick, C.T. Kelley, K.R. Kavanagh, Solution of a Groundwater Flow Problem with Implicit Filtering, Optimization and Engineering, (3)189-199 (2002)

4. T.D. Choi, O.J. Eslinger, P. Gilmore, A. Patrick, and C.T. Kelley, IFFCO: Implicit Filtering For Constrained Optimization, Version 2, Tech-Report, CRSC-TR99-23 NC State University (1999)

5. S.M. Gorelick, A Review of Distributed Parameter Groundwater Management Modeling Methods, Water Resources Research 19(2) 305-319 (1983)

6. K.R. Kavanagh (Fowler), C.T. Kelley, C.T. Miller, Mark S. Reed, C.E. Kees, J.P. Reese, M.W. Farthing, and R.W. Darwin, Solution of a well field Design Problem with Implicit Filtering, To Appear in Optimization and Engineering (203)

7. G.P. Karatzas and G. F. Pinder, Groundwater Managemendt Using Numerical Simulation and Outer Approximation Method for Global Optimization, Water Resources Research, 29(10) 3371-3378, (1986)

8. C.T. Kelley, Iterative Methods for Optimization, SIAM series in Frontiers in Applied Mathematics (18), Philadelphia (1999)

9. Daene C. McKinney and Min-Der Lin, Approximate Mixed-Integer Nonlinear Programming Methods for optimal Aquifer Remediation Design Water Resources Research, 3193) 731-740, (1995) 
10. A.S. Meyer, C.T. Kelley, and C.T. Miller, Optimal Design for Problems Involving Flow and Transport in Saturated Porous Media, Advances in Water Resources, (12) $1233-1256$ (2002).

11. A.S. Meyer, C.T. Kelley, and C.T. Miller, Electronic Supplement to "Optimal Design for Problems Involving Flow and Transport in Saturated Porous Media", Advances in Water Resources, (12) 1233-1256 (2002).

12. Ann E. Mulligan and David P. Ahlfeld, Advective Control of Groundwater Contaminant Plumes: Model Development and Comparison to Hydraulic Control, Water Resources Research, 35(8) 2285-2294 (1999)

13. Liang-Cheng Chang, Christine Shoemaker, and Philip L.F. Lui, Optimal TimeVarying Pumping Rates For Groundwater Remediation: Application of a Constrained Optimal Control Algorithm, Water Resources Research, (28) 3157-3173 (1992).

14. Brian J. Wagner, Recent Advances in Simulation-Optimization Groundwater Management Modeling, U.S. National Report to IUGG, 1991-1994.

15. Chunmiao Zheng and P.Patrick Wang, MT3DMS: A Modular Three-Dimensional Multispecies Transport Model for Simulation of Advection, Dispersion, and Chemical Reactions of Contaminants in Groundwater Systems; Documentation and User's Guide, 1999.

16. Chunmiao Zheng, Mary C. Hill, and Paul A. Hsieh, MODFLOW2000, The U.S.G.S Survey Modular Ground-Water Model User Guide to the LMT6 Package, the Linkage With MT3DMS for Multispecies Mass Transport Modeling, User's guide, 2001. 